\title{
Lateral Manipulation of Single Adsorbates and Substrate Atoms With the Scanning Tunneling
Microscope
}

G. Meyer and K.H. Rieder

\section{Introduction}

The stability and precision of modern scanning-tunneling-microscope (STM) systems allow positioning of the tip on a subnanometer scale. This advancement has stimulated diverse efforts on surface modifications in the nanometer and even atomic range, as recently reviewed by Avouris. ${ }^{1}$ The lateral movement of individual adatoms and molecules in a controlled manner on solid surfaces and the construction of structures on a nanoscale were first demonstrated by Eigler ${ }^{2}$ and collaborators at $4 \mathrm{~K}$. The reason for operating the STM at low temperatures (apart from increased stability and sensitivity of the STM setup itself) is the necessity to freeze the motion of single adsorbates, which are very often mobile at ambient temperatures. By selecting strongly bonded adsorbate/substrate combinations and large molecules, it was possible to extend the lateral manipulation technique even to room temperature. ${ }^{3,4}$ In the case of large molecules, not only their translational motion but also internal flexure of the molecule during the positioning process must be considered. ${ }^{3}$ In general, different physical and chemical interaction mechanisms between tip and sample can be exploited for atomic-scale manipulation. We will concentrate in the following on lateral manipulation where solely the forces that act on the adsorbate because of the proximity of the tip are used. This means that long-range van der Waals and short-range chemical forces can be used to move atoms or molecules along the surface. No bias voltage or tunneling current is necessary. Apart from this technique, additional advances using the effects caused by the electric field generated by the bias voltage between tip and sample and by the current flowing through the gap region can be used for atomic or molecular modification.

A few experimental prerequisites must be taken into account if one wants to perform manipulation on single atoms or small molecules. Especially when working on metal surfaces, it is important to operate the STM at low temperatures since single metal atoms and weakly bonded adsorbates are mobile at ambient temperatures. To freeze this motion, one usually must cool even below liquidnitrogen temperature. Furthermore the manipulation of single atoms/molecules means working at extremely low coverage - that is, using only a few atoms (for example 100) for a long time (several days). This requires both high cleanliness and the ability to deposit small amounts of adsorbates in a controlled manner. One can achieve these results surrounding the whole STM by a liquidhelium radiation shield and depositing small amounts of adsorbates through a small hole in the shield. Finally a flexible and precise tip-positioning procedure and--when creating and investigating nanostructures - a high stability of the whole system are necessary.

Figure 1a shows a schematic STM setup for manipulation. If the apex of the tunneling tip is far away from the adsorbate, only long-range forces like van der Waals forces are exerted on the adsorbate. When the tip comes close enough to the adsorbate, one enters the regime of short-range chemical forces. Since both distance and orientation of this chemical bond between tip apex and adsorbate can be controlled by adjusting the tip position, this situation is termed a "tunable chemical bond." The procedure for lateral manipulation is remarkably simple and appears in Figure $1 b$ : In the STM imaging process, the tip is scanned at distances of a few atomic diameters above the surface (a) and-in the constant current mode-follows contours of constant local electronic density of states. ${ }^{5}$ For manipulation the tip is brought close to the adspecies (b) by reducing the tunneling resistance. Then the tip is moved parallel to the surface (c) to a predetermined place. The particle is thereby pulled (or pushed) to the desired location (d). The tip is then drawn back to the scanning distance (e), and a new STM picture is scanned to check the result of the manipulation. The magnitude of the force acting on an atom/molecule is not directly accessible to the STM. The force is however a function of the distance between tip and adsorbate. Controlling the distance thus gives a measure of the exerted force. Moreover since the tunneling current at low voltages is proportional to the bias voltage and depends exponentially on the distance, ${ }^{5}$ the tunneling resistance can be used as a rough measure to control the force acting on the adsorbate. This results in a threshold of the tunneling resistance, below which adsorbates can be moved. The indirect control of the force results in the consequence that the threshold values of the tunneling resistance depend significantly on the tip structure. Careful and reproducible tip preparation is therefore an important factor.

\section{Manipulation of Adsorbates}

Several examples of manipulation discussed in the following have been performed on a stepped metal surface: the copper (211) surface. The top view of a sphere model of the clean $\mathrm{Cu}(211)$ substrate appears in Figure 2: The surface consists of (111) terraces separated by (100) single step facets. The $\mathrm{Cu}$ atoms are represented by the small spheres with the topmost $\mathrm{Cu}$ atoms shaded brightest. The dimensions of the unit cell 

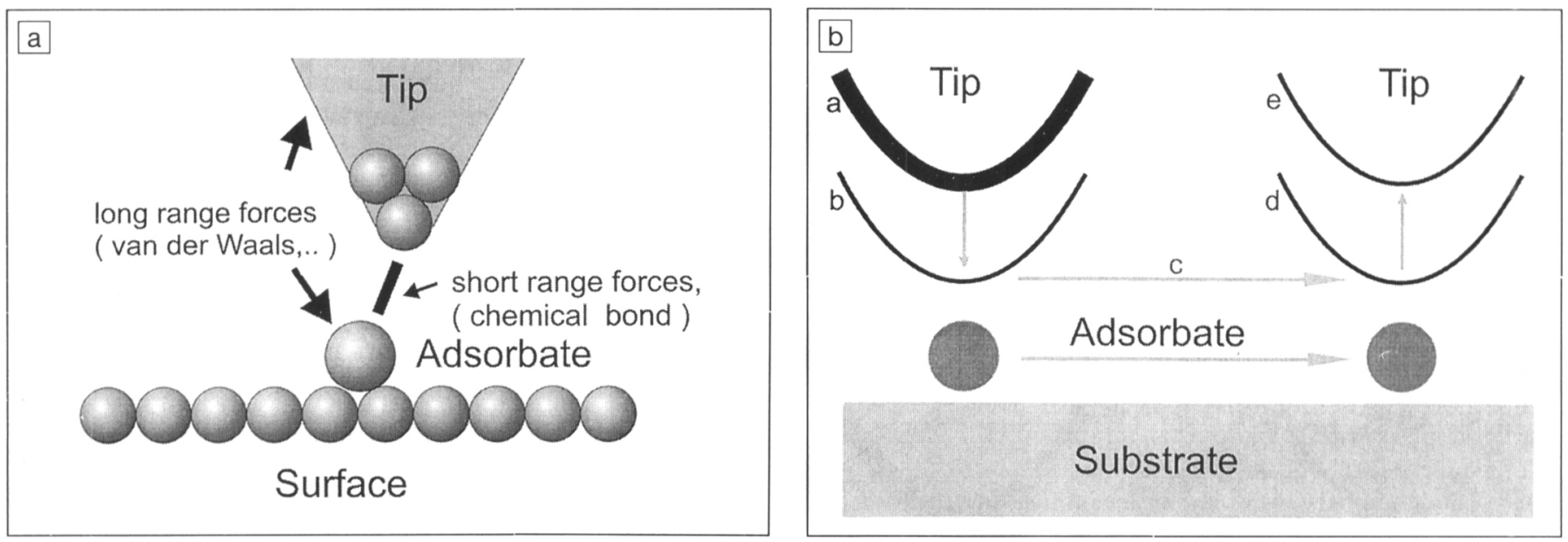

Figure 1. (a) Schematic scanning-tunneling-microscopy (STM) manipulation setup: The apex of the tunneling tip is close to the adsorbate. Long-range forces and short-range chemical bonding forces help move the adsorbate around the surface. (b) Working steps for lateral manipulation (for details see text).

are $a_{1}=0.255$ and $a_{2}=0.625 \mathrm{~nm}$. Significant interest in stepped surfaces has surfaced in the past years, especially in studies on catalysis and crystal growth. This is because adsorbates usually have a higher binding energy at the intrinsic step edges. Therefore adsorption is mainly confined to sites on top or down at the step edges. This highly anisotropic character of the stepped surfaces can also be observed and exploited in lateralmanipulation experiments. Moving adsorbates along an intrinsic step edge will yield different results than moving adsorbates across a step edge. More important the movement of the adsorbate can be confined along the step edges.

The first adsorbate we studied on this surface was carbon monoxide. On metal surfaces, $\mathrm{CO}$ molecules at low coverage usually adsorb upright or tilted in on-top positions (directly on top of a surface atom) with the carbon atoms close to the metal substrate atoms. ${ }^{6}$ On $\mathrm{Cu}(211)$ the CO molecules occupy top sites over the $\mathrm{Cu}$ atoms forming the ridges of the regular steps. ${ }^{7}$ We could determine this location via registry information by laterally manipulating $\mathrm{CO}$ molecules and single $\mathrm{Cu}$ atoms close to each other. ${ }^{8} \mathrm{We}$ employed lateral manipulation of $\mathrm{CO}$ molecules to form nanostructures at 30 $40 \mathrm{~K}$. Their buildup and perfection suffered from frequent irregular diffusive jumps of the $\mathrm{CO}$ molecules. "In Figures $3 a-3 c$, we show the formation of the letter " $\mathrm{X}$ " at $15 \mathrm{~K}$ where diffusive motion is practically quenched so that nanostructures form with perfect precision. The CO molecules appear as single depressions in the images located on top of

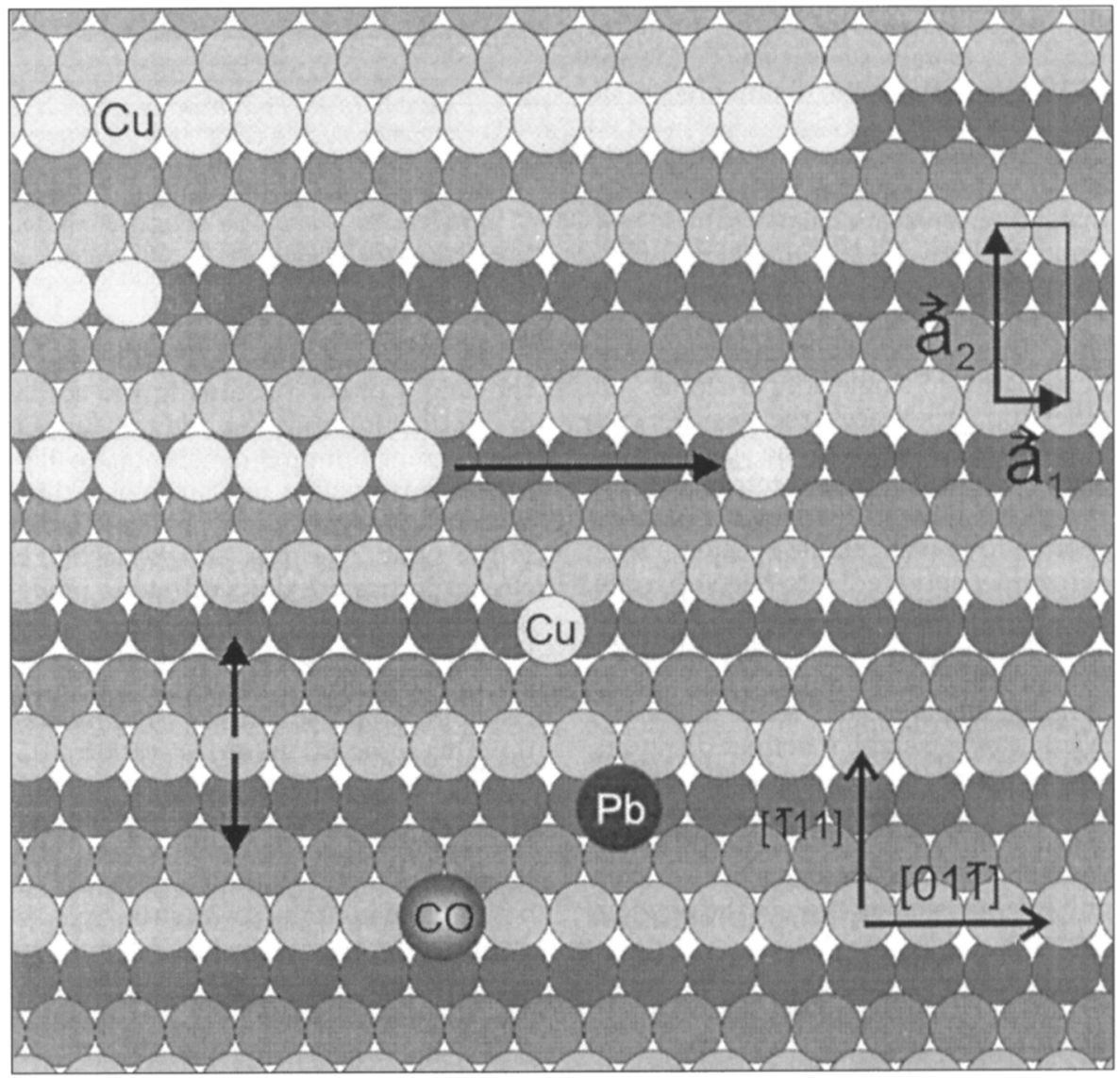

Figure 2. Sphere model of the Cu(211) surface; the unit cell is indicated. Part of a higher positioned defect terrace is plotted to illustrate the detachment of native $\mathrm{Cu}$ atoms from kink sites. The locations of single $\mathrm{Cu}$ atoms, $\mathrm{Pb}$ atoms, and CO molecules as determined from manipulation and coadsorption experiments also appear. The arrows indicate the extraction of kink and step-edge atoms. 
the intrinsic step edges. (In simplistic terms, they appear as depressions because the bonding between the $\mathrm{CO}$ molecule and the metal surface reduces the density of states at the Fermi level, which is a major contributor to the tunneling current.) No changes in either the structure of the letter " $\mathrm{X}$ " or its surroundings appeared an hour after its buildup, proving the stability of nanostructures formed even with weakly chemisorbed species like CO. The fact that with our STM we scanned exactly the same area of the surface 60 minutes later is proof of the extremely good mechanical stability of our instrument at these low temperatures. The lateral drift is of the order of $0.1 \mathrm{~nm} / \mathrm{h}$. (At higher temperatures, it is appreciably larger.) Owing to the pronounced anisotropy of the $\mathrm{Cu}(211)$ substrate, manipulation of the CO molecules along the step edges proceeds readily whereas manipulations perpendicular to the steps are almost impossible. In sliding single $\mathrm{CO}$ molecules parallel to the step edges, a reduction of the tunneling resistance to values between $600 \mathrm{k} \Omega$ and $1 \mathrm{M} \Omega$ was sufficient to obtain precise lateral movements.

As a second example, we present the lateral manipulation of single $\mathrm{Pb}$ atoms that have been evaporated at sample temperatures between 20 and $80 \mathrm{~K}$. At these low temperatures, $\mathrm{Pb}$ is adsorbed in the fivefold adsites located down at the step edge. This was deduced from registration relative to single copper atoms. ${ }^{8} \mathrm{On}$ other copper surfaces, the incorporation of single $\mathrm{Pb}$ atoms into the substrate has been observed at room temperature. ${ }^{10}$ However temperatures used here are low enough to prevent single $\mathrm{Pb}$ atoms from being incorporated into the substrate. Figure $3 \mathrm{~d}$ shows four $\mathrm{Pb}$ atoms positioned by lateral manipulation to form the corners of a rectangle. The distance of the $\mathrm{Pb}$ atoms parallel to the step edges is six nearest-neighbor copper distances, and the overall height of $\mathrm{a} \mathrm{Pb}$ atom is $0.1 \mathrm{~nm}$. The threshold tunneling resistance to manipulate single $\mathrm{Pb}$ atoms varied between 400 and $600 \mathrm{k} \Omega$, which is only slightly smaller than in the previous example.

\section{Pulling, Pushing, and Sliding of Adsorbates}

As explained before, the lateral manipulation proceeds by bringing the tip close to the adsorbate through reduction of the tunneling resistance and then, at constant tunneling resistance by moving parallel to the surface with the atom following the tip. We now discuss the details of the movement of the adsorbate during
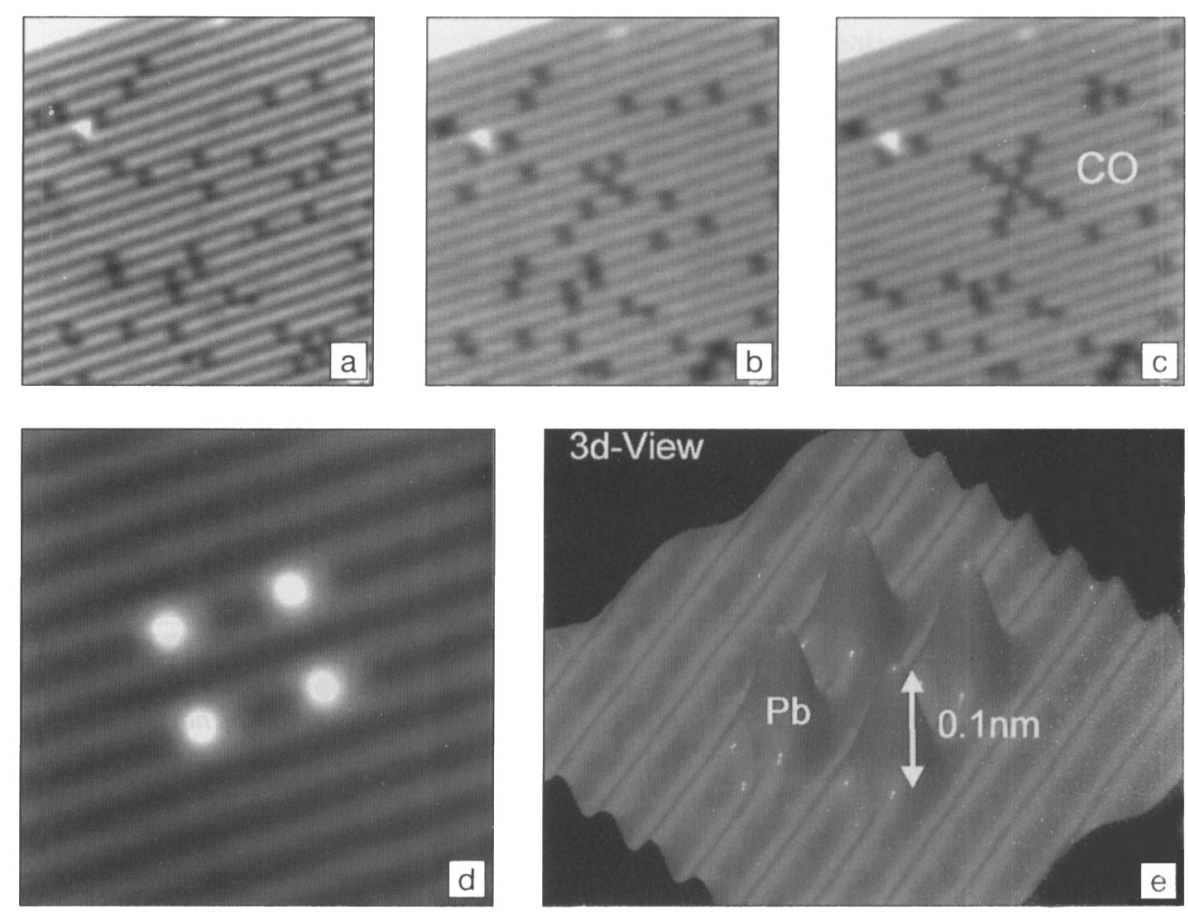

Figure 3. $(a-c)$ Formation of letter " $X$ " by controlled lateral manipulation of CO molecules on Cu(211) at $15 \mathrm{~K}$ using an STM. The size of each image is $13.5 \mathrm{~nm} \times 13.5 \mathrm{~nm}$. (d) Regular rectangle formed by lateral manipulation of single $\mathrm{Pb}$ atoms. The distance along the step edges corresponds to six Cu nearest neighbors. The height of each Pb atom is $0.1 \mathrm{~nm}$. Image size is $6 \mathrm{~nm} \times 6 \mathrm{~nm}$. (e) Corresponding perspective view.

the manipulation process. Figure 4 shows the height of the tip during the actual manipulation process while the tunneling current remains constant. ${ }^{11}$ In Figure $4 \mathrm{a}$, a tunneling resistance of $120 \mathrm{k} \Omega$ is used, and the tip is moving from the left to the right. The steps in the tip height clearly indicate a discontinuous movement of the $\mathrm{Pb}$ atom. This means that the $\mathrm{Pb}$ atom is jumping from one adsorption site to the next. Furthermore when the $\mathrm{Pb}$ atom jumps, it is always behind the tip. This indicates an attractive force between the tip and the atom. Therefore the $\mathrm{Pb}$ atom is pulled by the tip. (The first jump is in the opposite direction compared to the other jumps and is due to the $\mathrm{Pb}$ atom jumping toward the tip apex when it first approaches the atom.) Figure $4 \mathrm{~b}$ shows that, by reducing the tunneling resistance to $43 \mathrm{k} \Omega$, the $\mathrm{Pb}$ atom continuously follows the tip and no jumps occur. This mode is called sliding. Since the force between a metal tip apex and a metal atom is always attractive in the tunneling regime, one must use a different adsorbate to show the effect of repulsive forces. Figure $4 \mathrm{c}$ shows the tip height during manipulation of a $\mathrm{CO}$ molecule along an intrinsic $\mathrm{Cu}(211)$ step edge. The CO molecule appears as a depression in this image. One can see that during movement of the tip from left to right, the jumps occur always before the tip reaches the center of the CO molecule. Therefore the molecule is always in front of the tip, which now indicates a repulsive force. In this case, the molecule is pushed by the tip. Because of the anisotropy of the substrate, the tip will not lose the molecule during pushing as it is always bonded to the step edge. In general such detailed measurements, also including differently oriented tips, will not only help to improve the reliability of the manipulation technique but will also provide valuable information for theoretical calculations of the repositioning mechanisms. ${ }^{12}$

\section{Manipulation of Substrate Atoms}

While in the previous sections we discussed the lateral manipulation of adso! bates on the surface, we will now show that even strongly bound substrate atoms can be manipulated. ${ }^{8,13}$ Figures $5 a-5 c$ show the extraction of three $\mathrm{Cu}$ atoms out of the intrinsic step edges of the $\mathrm{Cu}(211)$ surface. The direction and length of the tip movement are indicated 
by the black arrow in Figure 5a. In Figure $5 c$, the extracted $\mathrm{Cu}$ atoms have been moved further along the step edges to show the three vacancy sites, indicated by the three white arrows. As one might expect, it is also possible to fill a vacancy again with an adatom. There we point out a new, interesting observation: A larger force is needed to bring the $\mathrm{Cu}$ atoms into the vacancies than to move them along the close-packed rows. This points to a local buildup of an EhrlichSchwoebel barrier ${ }^{14}$ and raises the hope that, in the future, quantitative measurements on local barriers will be possible.

Apart from the above described extraction of substrate atoms out of stepedge sites of the $\mathrm{Cu}(211)$ surface, we have demonstrated a complete set of manipulation procedures with which atom-byatom structuring of the surface is possible. ${ }^{13}$ Substrate atoms located in fivefold, sixfold, and even sevenfold coordinated sites can be manipulated. In all cases, only attractive forces between the substrate atoms and the tip were used. Effective-medium theory calculations of surface defects on $\mathrm{Cu}(111)$ show that the activation energy for diffusion of atoms out of intrinsic steps onto the terrace amounts to $0.770 \mathrm{eV} .^{15} \mathrm{~A}$ few factors might help surmount this high energy barrier. One is that this energy constitutes only an upper bound because the interaction with the tip apex will pull the atom away from the surface, and thus reduce the magnitude of lateral interactions between the surface and the atom. ${ }^{2}$ Second, temperature will assist in crossing the barrier. Moreover since no direct way of determining the chemical composition of the tip apex exists, it is possible that tungsten atoms reside at the tip apex. These atoms would exert significantly higher manipulation forces than copper atoms. ${ }^{16}$

One technical problem to be solved for manipulation of native substrate atoms is how to prepare a tip stable enough to resist the high attractive forces during the extraction of single atoms from highly coordinated substrate sites. Until now this has occurred through usual trialand-error techniques. After successfully preparing a tip capable of a certain manipulation procedure, one can repeat the process reliably many times. This leads to the possibility that extended parts of the substrate can be restructured in an atom-by-atom way. We demonstrate this in Figures $5 d-5 g$, in which more than 30 atoms were laterally manipulated so that a rectangular area on the terrace below is laid open. Such operations will be important in cases where subsurface de-

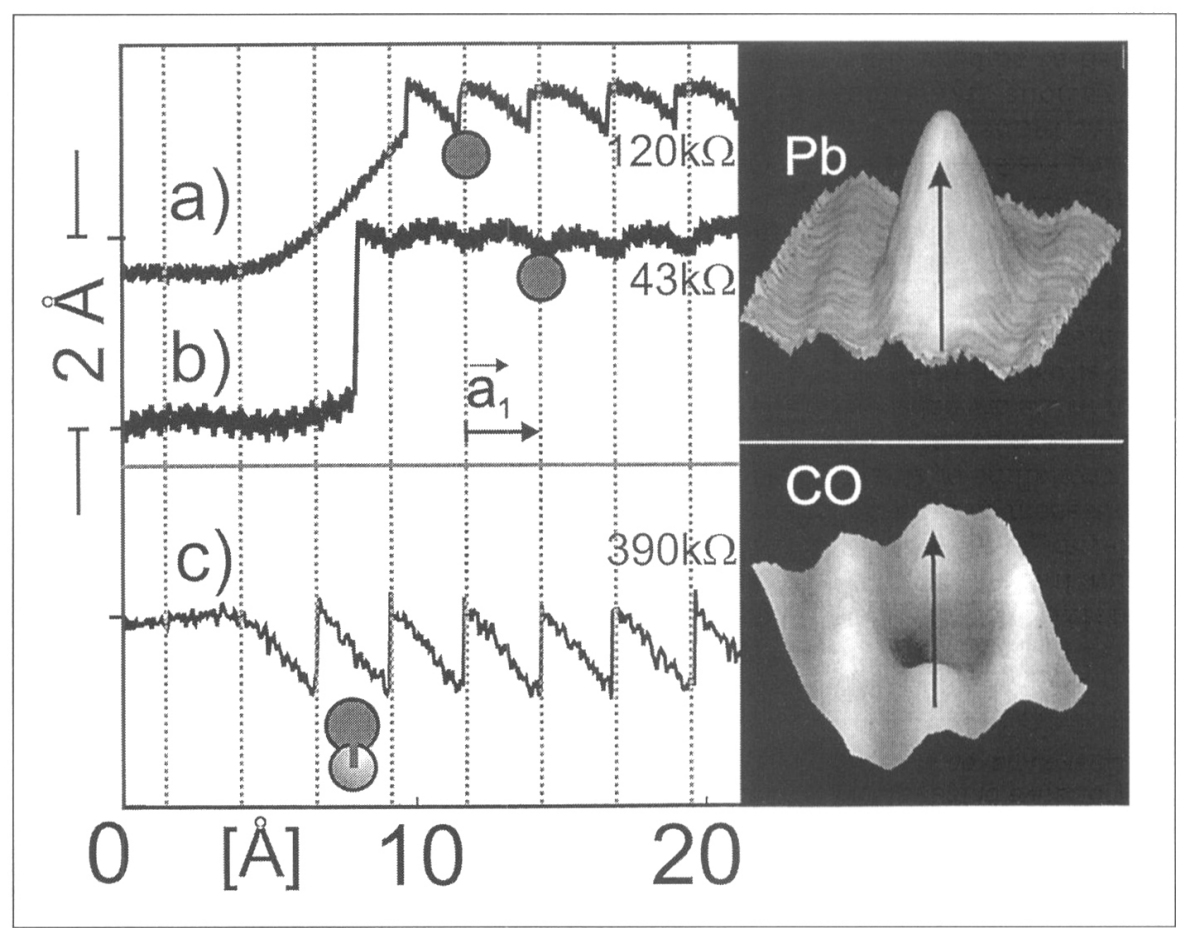

Figure 4. The left part of the figure shows STM tip-height curves during pulling/sliding of $\mathrm{a} P \mathrm{~Pb}$ atom ( $a, b$ ) and pushing of a CO molecule (c). The tip movement is from left to right. Images of the adparticles appear on the right. The steps in the tip height observed in (a) and (c) indicate that the atom/molecule jumps from one adsorption site to the next during manipulation while in $(b)$ the atom continuously follows the tip.

fects should be identified, removed, or deliberately created.

\section{Conclusion and Outlook}

In summary we have shown that single adatoms and molecules can be laterally manipulated on a surface in a controlled manner. This allows the buildup of nanostructures with atomic-scale precision. Moreover detailed information can be gathered on the behavior of adspecies during the manipulation process, leading to the distinction of three movement modes: pulling, sliding, and pushing. The extraction of single native substrate atoms in a controlled manner from differently coordinated sites of the substrate demonstrates that the lateral-manipulation technique is not restricted to adsorbates. Apart from use of the substrate atoms - and the corresponding vacancies - to build prototypic nanostructures, the manipulation of substrate atoms will be important in gathering information about subsurface defects. Since atoms may be moved into vacancy sites with foreign species, atom-by-atom surface alloying will become possible. In the future, further in- sight into the manipulation process may be achieved through use of using an atomic force microscope to directly measure the forces during manipulation. More important this would also facilitate work on insulating substrates. By optimizing the substrate temperature for a certain manipulation process, one can more strongly manipulate bound adsorbates. One might also imagine an indirect manipulation process where adsorbates are manipulated not in direct contact with the tip but through special adspecies.

\section{Acknowledgment}

This project was financed by the Deutsches Forschungsgemeinschaft, Contract No. RI 472/3-1.

\section{References}

1. P. Avouris, Acc. Chem. Re's. 28 (1995) p. 95 and references therein.

2. J.A. Stroscio and D.M. Eigler, Science 254 (1991) p. 1319; M.F. Crommie, C.P. Lutz, and D.M. Eigler, ibid. 262 (1993) p. 218

3. T.A. Jung, R.R. Schlittler, H. Tang, C. Joachim, and J.K. Gimzewski, ibid. 271 (1991) p. 181. 

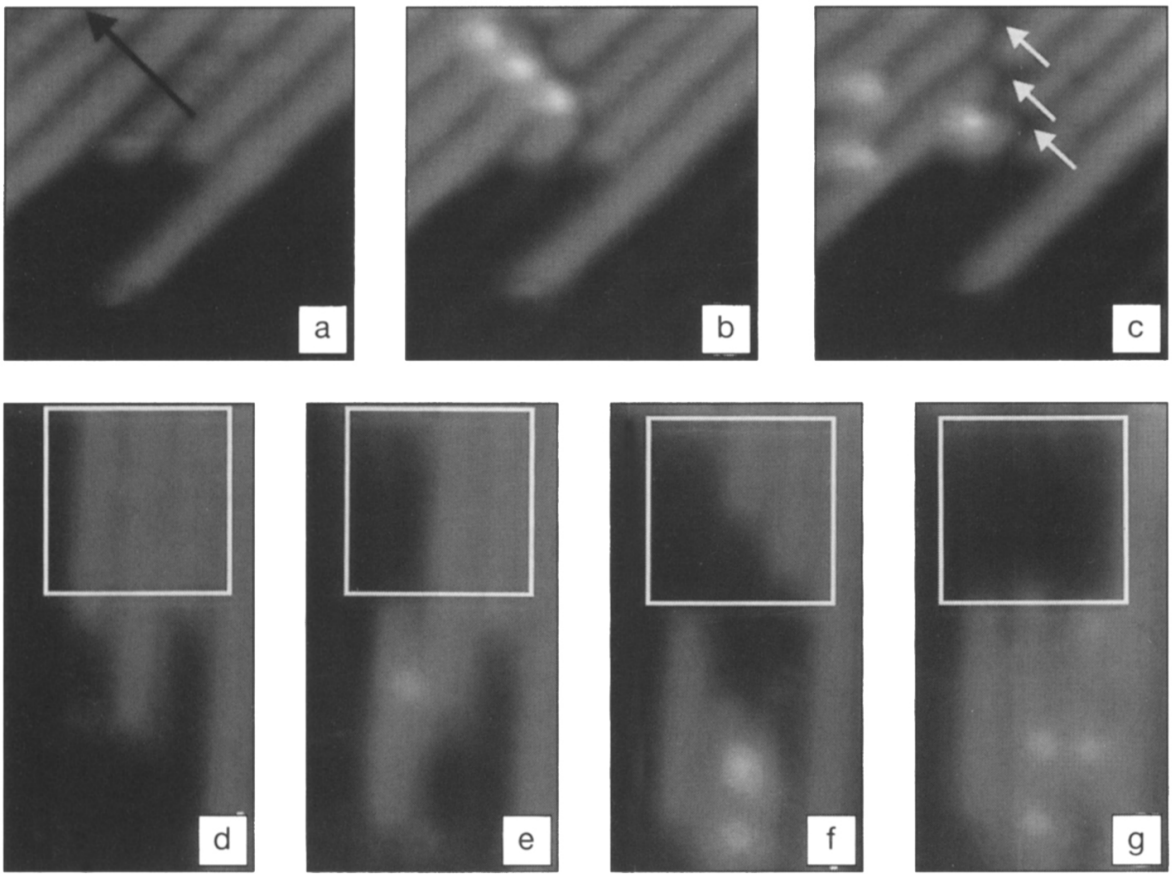

Figure 5. (a) Surface of $\mathrm{Cu}(211)$ with a defect step separating two terraces. (b) Extraction of three Cu atoms out of the intrinsic step-edge sites along a line indicated by the black arrow in (a). (c) The Cu atoms move along the step edges to expose the three vacancy sites indicated by the three white arrows. $(d-g)$ Extended substrate restructuring on a $\mathrm{Cu}(211)$ surface. From the area indicated by the square in (d), more than 30 atoms of the upper terrace are displaced to open up a rectangular area on the lower terrace as shown in (g). The sample bias voltage was $47 \mathrm{mV}$. The current used for manipulation was $130 \mathrm{nA}$ and that used for imaging was $1.35 n A$.

4. P.H. Beton, A.W. Dunn, and P. Moriarty, Surf. Sci. 361/362 (1996) p. 878; Y.W. Mo, Sci(')ice 261 (1993) p. 886.

5. J. Tersoff and D.R. Hamann, Phys. Rev. Lett. 50 (1983) p. 1998.

6. S. Ishi, Y. Ohno, and B. Viswanathan, Surf. Sci. 161 (1985) p. 349.

7. G. Meyer, B. Neu, and K.H. Rieder, Chem. Phys. Lett. 240 (1995) p. 379.
8. G. Meyer, S. Zöphel, and K.H. Rieder, Phys. Rev. Lett. 77 (1996) p. 2113.

9. G. Meyer, B. Neu, and K.H. Rieder, Appl. Phys. A 60 (1995) p. 343.

10. C. Nagl, O. Haller, E. Platzgummer, M. Schmid, and P. Varga, Surf. Sci. 321 (1994) p. 237.

11. L. Bartels, G. Meyer, and K.H. Rieder, Phys. Rev. Lett. 79 (1997) p. 697.
12. X. Bouju, Ch. Girard, H. Tang, C. Joachim, and L. Pizzagalli, Phys. Rev. B 55 (1997) p. 16498; S. Ciraci, E. Tekman, A. Baratoff, and I.P. Batra, ibid. 46 (1992) p. 10411

13. G. Meyer, L. Bartels, S. Zöphel, E. Henze, and K.H. Rieder, Phys. Rev. Lett. 78 (1997) p. 1512.

14. S.C. Wang, and G. Ehrlich, ibid. 70 (1993) p. 41; R.L. Schwoebel, J. Appl. Phys. 40 (1969) p. 614 .

15. P. Stoltze, I. Phys. Cond. Matter 6 (1994) p. 9495 .

16. E. Koetter, D. Drakova, and G. Doyen, Surf. Sci. 331-333 (1995) p. 679.

G. Meyer received a $\mathrm{PhD}$ degree from the University of Hannover, Germany, in highresolution low-energy-electron-diffraction studies on epitaxial growth. He served as a postdoctoral student at IBM Research, Yorktown Heights, New York, USA, studying scanning tunneling microscopy and atomic force microscopy. Meyer also performed postdoctoral work at Max-Planck-Institut für Strömungsforschung Göttingen. Since 1992 he has worked in the research group of K.H. Rieder at the Free University of Berlin. Meyer's research focuses on lowtemperature scanning tunneling microscopy and atomic/molecular manipulation. He can be reached at the following $e$-mail address: gmeyer@physik.fu-berlin.de.

\section{K.H. Rieder received a PhD degree from} the University of Vienna. His work experience includes research on reactor materials at the Austrian Reactor Center Seibersdorf; work on n-scattering, and infrared and Raman spectroscopy at MPI für Festkörperforschung in Stuttgart; and time at IBM Rüschlikon focusing on atom-beam diffraction. Since 1986 he has served as a professor at the Free University of Berlin. Rieder's research focuses on atom-beam scattering, scanning tunneling microscopy, and mechanic and dynamic properties of magnetic thin films.

\section{Please contact MRS at its new location:}

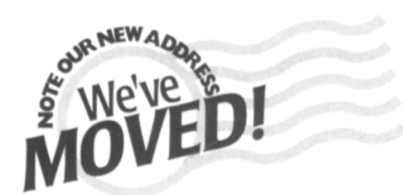

Old Address:

9800 McKnight Road, Pittsburgh, PA 15237

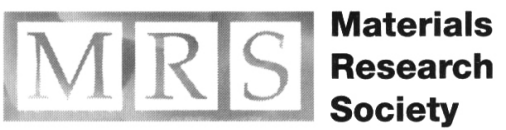

506 Keystone Drive, Warrendale, PA 15086-7573 USA
Phone (operator): 412-779-3003

Phone (automated attendant): 412-779-3004

Advertising \& Exhibits: 412-779-8312

Fax: 412-779-8313 